\title{
Tree-Based Key Distribution Patterns
}

\author{
Jooyoung Lee ${ }^{1}$ and Douglas R. Stinson ${ }^{2}$ \\ 1 Department of Combinatorics and Optimization \\ 2 School of Computer Science, University of Waterloo, \\ Waterloo, Ontario, Canada N2L 3G1 \\ \{j3lee, dstinson\}@uwaterloo.ca
}

\begin{abstract}
We revisit a key agreement scheme presented by Leighton and Micali [11], generalize the scheme, and present a new framework of tree-based key distribution pattern (TKDP). We presents a method of constructing TKDPs from cover-free families. We show the existence of TKDPs by probabilistic method. We can reduce the upper bounds on the minimum number of rows of $(t, w, \mathcal{T})$-TKDPs, which are obtained from probabilistic methods, asymptotically by a factor of $w$ as compared to Leighton and Micali's schemes by choosing optimal trees $\mathcal{T}$ instead of chains.
\end{abstract}

Keywords: key predistribution, cover-free family.

\section{Introduction}

In our model, the network consists of a trusted authority (TA) and a set of users $\mathcal{U}=\left\{U_{1}, \ldots, U_{n}\right\}$. Let $2^{\mathcal{U}}$ denote the collection of all subsets of users. $\mathcal{P} \subseteq 2^{\mathcal{U}}$ and $\mathcal{F} \subseteq 2^{\mathcal{U}}$ will denote the collection of privileged subsets and the collection of forbidden subsets, respectively. $\Gamma=(\mathcal{P}, \mathcal{F})$ is called an access structure. Roughly speaking, a key predistribution scheme (KPS) with an access structure $\Gamma$ is a method for the TA to distribute secret shares to each user in the network, so that any user in a privileged subset $P$ can easily compute their group key $K_{P}$, while any coalition $F \in \mathcal{F}$ disjoint from $P$ can compute $K_{P}$ only with negligible probability. In this paper, we will consider an access structure $\Gamma_{(t, w)}=(\mathcal{P}, \mathcal{F})$ such that $\mathcal{P}=\{P \subseteq \mathcal{U}:|P|=t\}$ and $\mathcal{F}=\{F \subseteq \mathcal{U}:|F|=w\}$. Thus any $t$ users can compute a common key while any coalition of at most $w$ users can obtain no information on the key. (Such KPSs are called $(t, w)$-KPSs.) One method of constructing KPSs uses key distribution patterns (KDPs), due to Mitchell and Piper [13. We will extend the study of KDPs by allowing hashed shares of information in the generation of secret keys.

\subsection{Motivation: Leighton and Micali Scheme}

This work is motivated by a key agreement scheme proposed by Leighton and Micali [1]. Here we briefly describe their scheme (with slight modification):

1. The TA publishes a (one-way) hash function

$$
h:\{0,1\}^{l} \longrightarrow\{0,1\}^{l},
$$

where $l$ is a secret key length used for symmetric encryption. 
2. For each user $U_{j}$, a public sequence $A_{j} \in\{0, \ldots, L-1\}^{b}$ is chosen in a uniformly random way, where $b$ and $L$ are positive integers. The public sequences act as public identities of users, and they comprise a $b \times n$ matrix $M=\left(A_{1}^{T}|\cdots| A_{n}^{T}\right)=\left(\alpha_{i, j}\right)$.

3. The TA chooses random seeds $s_{i}=s(i, 0) \in\{0,1\}^{l}$ for $i=1, \ldots, b$ and computes hashed shares $s(i, \alpha)=h^{\alpha}\left(s_{i}\right)$ for $\alpha=1, \ldots, L-1$. $\left(h^{\alpha}(s)\right.$ indicates applying the function $h$ iteratively $\alpha$ times on the input $s$.)

4. User $U_{j}$ receives a set of secret shares $s\left(i, \alpha_{i, j}\right)$ for $i=1, \ldots, b$. Therefore, $b$ is the number of secret shares assigned to each user.

5. A pairwise key between $U_{j_{1}}$ and $U_{j_{2}}$ is defined to be

$$
K_{j_{1}, j_{2}}=s\left(1, \delta_{1}\right)+\ldots+s\left(b, \delta_{b}\right)
$$

where $\delta_{i}=\max \left(\alpha_{i, j_{1}}, \alpha_{i, j_{2}}\right)$. Addition is defined in $\left(\mathbb{Z}_{2}^{l},+\right)$.

We can easily derive a condition that a coalition of $w$ users, $U_{j_{1}^{\prime}}, \ldots, U_{j_{w}^{\prime}}$ obtain a hashed key between innocent users $U_{j_{1}}$ and $U_{j_{2}}$ as follows:

$$
\min \left(\alpha_{i, j_{1}^{\prime}}, \ldots, \alpha_{i, j_{w}^{\prime}}\right) \leq \max \left(\alpha_{i, j_{1}}, \alpha_{i, j_{2}}\right),
$$

for all $i=1, \ldots, b$. The authors of [1] computed the probability that a set of randomly chosen sequences protects pairwise keys against adversarial coalitions of size at most $w$. They claim that the probability becomes large enough when $w$ is about $(b / e \ln n)^{1 / 3}$.

Example 1.1. Suppose public sequences $A_{1}=(5,2,1), A_{2}=(2,3,1)$ and $A_{3}=$ $(1,3,3)$ are assigned to users $U_{1}, U_{2}$ and $U_{3}$, respectively. Then $U_{1}, U_{2}$ and $U_{3}$ receive key sets $\left\{h^{5}\left(s_{1}\right), h^{2}\left(s_{2}\right), h\left(s_{3}\right)\right\},\left\{h^{2}\left(s_{1}\right), h^{3}\left(s_{2}\right), h\left(s_{3}\right)\right\}$ and $\left\{h\left(s_{1}\right), h^{3}\left(s_{2}\right)\right.$, $\left.h^{3}\left(s_{3}\right)\right\}$, respectively. $U_{1}$ and $U_{2}$ can communicate with each other with key $K_{1,2}=h^{5}\left(s_{1}\right)+h^{3}\left(s_{2}\right)+h\left(s_{3}\right)$. An adversary $U_{3}$ can compute $h^{5}\left(s_{1}\right)$ and $h^{3}\left(s_{2}\right)$, but not $h\left(s_{3}\right)$; therefore the key $K_{1,2}$ is secure against $U_{3}$.

\subsection{Extension of Leighton and Micali Scheme}

We extend Leighton and Micali's scheme in three directions.

Pairwise Key to Group Key. Any $t$ users $U_{j_{1}}, \ldots, U_{j_{t}}$ can define their group key to be

$$
K_{j_{1}, \ldots, j_{t}}=s\left(1, \delta_{1}\right)+\ldots+s\left(b, \delta_{b}\right),
$$

where $\delta_{i}=\max \left(\alpha_{i, j_{1}}, \ldots, \alpha_{i, j_{t}}\right)$. In order for a coalition of $w$ users, $U_{j_{1}^{\prime}}, \ldots, U_{j_{w}^{\prime}}$, to obtain the group key, the following should occur:

$$
\min \left(\alpha_{i, j_{1}^{\prime}}, \ldots, \alpha_{i, j_{w}^{\prime}}\right) \leq \max \left(\alpha_{i, j_{1}}, \ldots, \alpha_{i, j_{t}}\right),
$$

for all $i=1, \ldots, b$.

Linear Ordering to Tree Ordering. Suppose that a matrix $M=\left(\alpha_{i, j}\right)$ of public sequences is determined. For each row of the matrix, a "seed" secret 


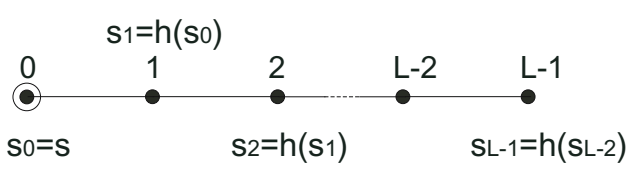

(a) Linear ordering

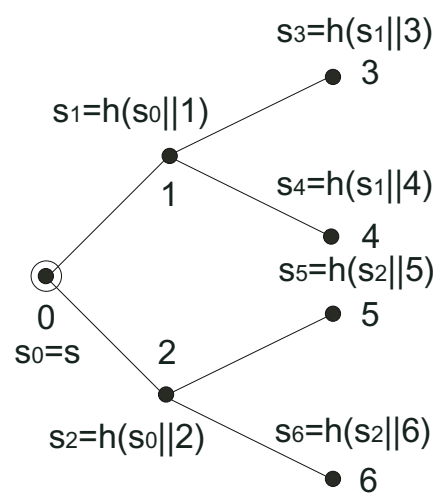

(b) Tree-based ordering

Fig. 1. Orderings of symbols

share is generated and each entry represents the hash depth applied to the seed. Therefore, we observe a hierarchy or an ordering between entries (symbols) in the sense that one information is enough to compute the other, but not conversely. For two symbols $\alpha_{1}$ and $\alpha_{2}$, we define $\alpha_{1} \leq \alpha_{2}$ if the information $\left(=h^{\alpha_{2}}(s)\right.$ ) associated with $\alpha_{2}$ is easily computed from the information $\left(=h^{\alpha_{1}}(s)\right)$ associated with $\alpha_{1}$. We can represent the ordering of symbols for each row as a chain structure (Fig. 1(a) , which coincides with the natural linear ordering of integers.

However we can also define nonlinear orderings based on rooted trees. For example, Fig. 1(b) shows an ordering of symbols based on a balanced binary tree of depth 2. Seven hashed shares are derived from a seed for each row, using (public) symbols of the tree in the hash computation. (The symbols are interpreted as binary sequences of a fixed length in the computation.)

Probabilistic Construction to Deterministic Construction. We can construct a matrix $M$ of public sequences in a deterministic way, instead of choosing random columns. For example, cover-free families, orthogonal arrays or ordered designs could be used. We also introduce a symbol $\infty$ as an entry of $M$, which means "no information". Based on a chain ordering, we can replace the largest symbol $L-1$ by $\infty$; since the corresponding hashed share $h^{(L-1)}(s)$ (for some seed $s$ ) can be computed by any other single user, it makes no contribution to secure communication. Therefore we would rather assign no information to the symbol in order to save memory storage per user.

\subsection{Other Related Works}

Blom [3] presented key predistribution schemes that require each user store $w+1$ keys to protect each pairwise key against any coalition attack of at most $w$ users. Blundo et al. 4] generalized Blom's scheme to allow for any group of size $t$ to establish its group key. For a group key to be secure against a coalition of $w$ users, each user has to store $\left(\begin{array}{c}t+w-1 \\ t-1\end{array}\right)$ keys. They also proved the key storage is 
information theoretically optimal. Mitchell and Piper [13 presented KPSs using key predistribution patterns, which involve very little arithmetic computation. The KDPs are equivalent to cover-free families, which are widely studied and generalized (for example, [17] and [18]). Such unconditionally secure key predistribution schemes are studied as special families of linear key predistribution schemes [14.

The efficiency of KPSs can be improved by choosing orthogonal arrays or perpendicular arrays as KDPs and then using resilient functions in the generation of keys [16. Recently, Attrapadung et al. [2] studied key predistribution schemes using key chains from pseudo-random sequence generators (PRSGs). In a network of $n$ users, each user stores $O\left(\frac{2^{n-1}}{n}\right)$ keys to establish group keys of any size, which are secure against any coalition attacks, based on the security of the PRSGs.

Since Eschenauer and Gligor's work 8], a series of papers on (pairwise) key predistribution for distributed sensor networks have been published ([5], 9], [10], [12], [15]). The main difference of these KPSs from conventional ones is that they allow certain pairs of nodes to share no common key. The attack model and the metric to evaluate the resiliency are also different, which are based on probabilistic analysis.

\subsection{Our Contributions}

By extending Leighton and Micali's scheme, we present a new framework of tree-based key distribution patterns (TKDPs). In section 3, we present a simple method of constructing TKDPs from cover-free families. In section 4, we show the existence of TKDPs by probabilistic methods, similar to [7]. Especially, the upper bounds on the minimum number of rows of $(t, w, \mathcal{T})$-TKDPs, which are obtained from probabilistic methods, are asymptotically $w$ times smaller than Leighton and Micali's schemes (with the same parameters), when we choose optimal trees $\mathcal{T}$ instead of chains. One advantage of TKDP-based KPSs over Leighton and Micali's schemes is smaller hash depths are applied to each secret seed, which means less computation is required.

\section{Tree-Based Key Distribution Pattern}

We are now prepared to present a framework of a tree-based key distribution pattern (TKDP). First, we define a rooted tree $\mathcal{T}$ on $L$ vertices, labeled from 0 to $L-1$. Especially, the root vertex is labeled by 0 . As we observed in the previous section, a rooted tree defines a partial ordering on $L$ vertices. We say $j_{1}<j_{2}$ if a vertex $j_{1}$ is an ancestor of $j_{2}$. For example, we observe that $0<3$ and $2<5$ in Fig. 1(b), But there is no relation between 1 and 2. We also define $j<\infty$ for every $j \in\{0, \ldots, L-1\}$. From now on, we will identify the set of users with the integers from 1 to $n$.

Definition 2.1. Let $\mathcal{T}$ be a rooted tree labeled by $\{0, \ldots, L-1\}$ and let $\Gamma=(\mathcal{P}, \mathcal{F})$ be an access structure. Let $M=\left(\alpha_{i, j}\right)$ be a $b \times n$ matrix with 
$\alpha_{i, j} \in\{0, \ldots, L-1\} \cup\{\infty\}$ for $1 \leq i \leq b$ and $1 \leq j \leq n$. We say that $M$ is a tree-based key distribution pattern $(\Gamma, \mathcal{T})$-TKDP $(b, n)$, provided that for every disjoint pair of $P \in \mathcal{P}$ and $F \in \mathcal{F}$, there exist $1 \leq i^{*}=i_{(P, F)}^{*} \leq b$ and $j^{*}=j_{(P, F)}^{*} \in P$ such that

1. $\alpha_{i^{*}, j} \leq \alpha_{i^{*}, j^{*}}$ for all $j \in P$, and

2. $\alpha_{i^{*}, j} \nless \leq \alpha_{i^{*}, j^{*}}$ for all $j \in F$.

The notation $(t, w, \mathcal{T})-T K D P(b, n)$ will denote a $\left(\Gamma_{(t, w)}, \mathcal{T}\right)-\operatorname{TKDP}(b, n)$.

KPS from TKDP. We construct a KPS with an access structure $\Gamma$ from a TKDP in the following manner:

1. The TA publishes a $(\Gamma, \mathcal{T})-\operatorname{TKDP}(b, n)$, denoted $M=\left(\alpha_{i, j}\right)$, a hash function

$$
h:\{0,1\}^{l_{1}+l_{2}} \longrightarrow\{0,1\}^{l_{1}},
$$

and a tree $\mathcal{T}$ on a set $\{0, \ldots, L-1\}$ of vertices.

2. For $1 \leq i \leq b$ and $1 \leq j \leq L-1$, the TA chooses random values $s_{i}=s(i, 0)$ of length $l_{1}$ and computes hashed shares $s(i, j)$ recursively based on the tree $\mathcal{T}$; if a vertex $j_{2}$ is a child of $j_{1}$, then $s\left(i, j_{2}\right)=h\left(s\left(i, j_{1}\right) \| j_{2}\right)$, where the label of each vertex is represented as a binary sequence of length $l_{2}$.

3. User $j$ receives secret shares $s\left(i, \alpha_{i, j}\right)$ for $1 \leq i \leq b$, where $s(i, \infty)=\emptyset$ by definition.

4. Let

$$
I_{P}=\left\{1 \leq i \leq b: \exists \bar{j} \in P \text { such that } \alpha_{i, j} \leq \alpha_{i, \bar{j}}, \forall j \in P\right\} .
$$

The key $K_{P}$ for a privileged set $P$ is defined to be

$$
K_{P}=\sum_{i \in I_{P}} s\left(i, \alpha_{i, \bar{j}}\right) .
$$

For a given $i \in I_{P}, \bar{j}$ is not necessarily unique, but $\alpha_{i, \bar{j}}$ is.

Any user $j \in P$ can compute every term in $K_{P}$ by using the function $h$, while a disjoint coalition $F \in \mathcal{F}$ cannot compute at least one term

$$
s\left(i_{(P, F)}^{*}, \alpha_{i_{(P, F)}^{*}, \bar{j}}\right),
$$

since $\alpha_{i_{(P, F)}^{*}, j} \not \leq \alpha_{i_{(P, F)}^{*}, \bar{j}}$ for all $j \in F$ (by Def. 2.1). In this case, we say row $i_{(P, F)}^{*}$ protects $P$ against $F$. If $l_{1}$ is the bit length of a secure key for a symmetric encryption, then we can say the KPS is secure with respect to the access structure $\Gamma$.

Remark 2.1. We assume a random oracle model for the function $h$ in the sense that we cannot obtain the value of $h(s)$ without query to the random oracle.

Remark 2.2. Each user should store $b l_{1}$ bits, or equivalently, $b$ secret keys. Therefore, we would like to have TKDPs that minimize $b$ with the other parameters fixed. 
Example 2.1. As we will see in Example 3.1.

$$
M=\left(\begin{array}{lllllll}
0 & 0 & 3 & 0 & 5 & 6 & 7 \\
1 & 0 & 0 & 4 & 0 & 6 & 7 \\
0 & 2 & 0 & 4 & 5 & 0 & 7 \\
1 & 2 & 3 & 0 & 0 & 0 & 7 \\
0 & 2 & 3 & 4 & 0 & 6 & 0 \\
1 & 0 & 3 & 4 & 5 & 0 & 0 \\
1 & 2 & 0 & 0 & 5 & 6 & 0
\end{array}\right)
$$

is a $\left(2,2, \mathcal{T}_{7}\right)-\operatorname{TKDP}(7,7)$, where $\mathcal{T}_{7}$ is a tree of depth 1 with 7 leaves (Fig. 2).

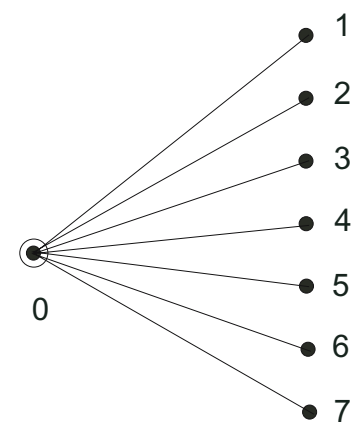

Fig. 2. Star-like tree: $\mathcal{T}_{7}$

The TA generates random secret seeds $s_{i}$ for $i=1, \ldots, 7$. Two users $U_{1}$ and $U_{2}$ (corresponding to the first two columns) receive sets of keys

$$
K_{1}=\left\{s_{1}, h\left(s_{2} \| 1\right), s_{3}, h\left(s_{4} \| 1\right), s_{5}, h\left(s_{6} \| 1\right), h\left(s_{7} \| 1\right)\right\},
$$

and

$$
K_{2}=\left\{s_{1}, s_{2}, h\left(s_{3} \| 2\right), h\left(s_{4} \| 2\right), h\left(s_{5} \| 2\right), s_{6}, h\left(s_{7} \| 2\right)\right\},
$$

respectively. For $P=\{1,2\}$, we have $I_{P}=\{1,2,3,5,6\}$. Both $U_{1}$ and $U_{2}$ can compute the group key

$$
K_{1,2}=s_{1}+h\left(s_{2} \| 1\right)+h\left(s_{3} \| 2\right)+h\left(s_{5} \| 2\right)+h\left(s_{6} \| 1\right) .
$$

An adversarial coalition of two users, say, $U_{3}$ and $U_{4}$, cannot compute $h\left(s_{5} \| 2\right)$ and $h\left(s_{6} \| 1\right)$. In fact, $K_{1,2}$ is secure against any coalition of size 2 .

\section{Cover-Free Families and TKDPs}

In this section, we will show some relations between cover-free families (or equivalently, conventional key distribution patterns) and TKDPs. A set system is a pair $(X, \mathcal{A})$, where $\mathcal{A}$ is a finite set of subsets of $X$, called blocks. 
Definition 3.1. A set system $(X, \mathcal{A})$ is called a $(t, w)$-cover-free family provided that, for any $t$ blocks $B_{1}, \ldots, B_{t} \in \mathcal{A}$ and any $w$ other blocks $A_{1}, \ldots, A_{w} \in \mathcal{A}$, we have

$$
\bigcap_{i=1}^{t} B_{i} \nsubseteq \bigcup_{j=1}^{w} A_{j} .
$$

A $(t, w)$-cover-free family will be denoted as a $(t, w)-\operatorname{CFF}(b, n)$ if $|X|=b$ and $|\mathcal{A}|=n$. Let $(X, \mathcal{A})$ be a $(t, w)-\operatorname{CFF}(b, n)$ such that $X=\left\{x_{1}, \ldots, x_{b}\right\}$ and $\mathcal{A}=\left\{A_{1}, \ldots, A_{n}\right\}$. The incidence matrix of $(X, \mathcal{A})$ is defined to be a $b \times n$ $(0,1)$-matrix $M=\left(\beta_{i, j}\right)$ such that $\beta_{i, j}=1$ if $x_{i} \in A_{j}$, and $\beta_{i, j}=0$, otherwise.

Now we can regard a cover-free family as a special case of TKDP, as seen in the following lemma.

Lemma 3.1. Let $L=1$, i.e., $\mathcal{T}$ consists of one vertex. Then $(t, w, \mathcal{T})-T K D P s$ are equivalent to $(t, w)$-cover-free families.

Proof. Let $M_{T}=\left(\alpha_{i, j}\right)$ be a $(t, w, \mathcal{T})$-TKDP $(b, n)$. Since $M_{T}$ is a $(0, \infty)$-matrix, we can define a $b \times n$ matrix $M_{C}=\left(\beta_{i, j}\right)$ such that

$$
\begin{cases}\beta_{i, j}=1, & \text { if } \alpha_{i, j}=0 \\ \beta_{i, j}=0, & \text { if } \alpha_{i, j}=\infty\end{cases}
$$

Then $M_{C}$ is the incidence matrix of a $(t, w)-\mathrm{CFF}(b, n)$. The converse is proved in a similar way.

There is a method of constructing TKDPs based on rooted trees of depth 1 from CFFs. (From now on, rooted trees of depth 1 are called star-like trees.) Let $\mathcal{T}_{n}$ be a star-like tree with root 0 and $n$ leaves (labeled from 1 to $n$ ).

Theorem 3.1. If there exists $a(t-1, w)-C F F(b, n)$, then there exists a $\left(t, w, \mathcal{T}_{n}\right)$ $\operatorname{TKDP}(b, n)$.

Proof. Let $M_{C}=\left(\beta_{i, j}\right)$ be the incidence matrix of a $(t-1, w)-\operatorname{CFF}(b, n)$. Then we can define a $b \times n$ matrix $M_{T}=\left(\alpha_{i, j}\right)$ as follows;

$$
\begin{cases}\alpha_{i, j}=0, & \text { if } \beta_{i, j}=1 ; \\ \alpha_{i, j}=j, & \text { if } \beta_{i, j}=0 .\end{cases}
$$

We claim that $M_{T}$ is a $\left(t, w, \mathcal{T}_{n}\right)-\operatorname{TKDP}(b, n)$.

The columns of $M_{C}$ and $M_{T}$ are indexed from 1 to $n$; the rows of $M_{C}$ and $M_{T}$ are indexed from 1 to $b$. Let $P=\left\{j_{1}, \ldots, j_{t}\right\}$ and $F=\left\{j_{1}^{\prime}, \ldots, j_{w}^{\prime}\right\}$ be disjoint subsets of columns. Since $M_{C}$ is the incidence matrix of a $(t-1, w)-\operatorname{CFF}(b, n)$, there exists a row $i$ such that $\beta_{i, j_{1}}=\ldots=\beta_{i, j_{t-1}}=1$, and $\beta_{i, j_{1}^{\prime}}=\ldots=\beta_{i, j_{w}^{\prime}}=$ 0 . Since $\alpha_{i, j_{1}}=\ldots=\alpha_{i, j_{t-1}}=0$, we have $\alpha_{i, j} \leq \alpha_{i, j_{t}}$ for every $j \in P$. On the other hand, we have $\alpha_{i, j^{\prime}} \nless \leq \alpha_{i, j_{t}}$ for every $j^{\prime} \in F$ since the symbols $\alpha_{i, j^{\prime}}$ represent distinct leaves of the tree. (In the context of KPS, the group key of $P$ contains a hashed share $h\left(s_{i} \| j_{t}\right)$ as a summand, while the coalition of $F$ cannot compute it.) 
Example 3.1. Let $t=w=2$ and $b=n=7$. Then we can construct a $\left(2,2, \mathcal{T}_{7}\right)$ $\operatorname{TKDP}(7,7)$, say $M_{T}$, from the incidence matrix $M_{C}$ of a $(1,2)-\operatorname{CFF}(7,7)$, where

$$
M_{C}=\left(\begin{array}{lllllll}
1 & 1 & 0 & 1 & 0 & 0 & 0 \\
0 & 1 & 1 & 0 & 1 & 0 & 0 \\
1 & 0 & 1 & 0 & 0 & 1 & 0 \\
0 & 0 & 0 & 1 & 1 & 1 & 0 \\
1 & 0 & 0 & 0 & 1 & 0 & 1 \\
0 & 1 & 0 & 0 & 0 & 1 & 1 \\
0 & 0 & 1 & 1 & 0 & 0 & 1
\end{array}\right) \quad \text { and } \quad M_{T}=\left(\begin{array}{lllllll}
0 & 0 & 3 & 0 & 5 & 6 & 7 \\
1 & 0 & 0 & 4 & 0 & 6 & 7 \\
0 & 2 & 0 & 4 & 5 & 0 & 7 \\
1 & 2 & 3 & 0 & 0 & 0 & 7 \\
0 & 2 & 3 & 4 & 0 & 6 & 0 \\
1 & 0 & 3 & 4 & 5 & 0 & 0 \\
1 & 2 & 0 & 0 & 5 & 6 & 0
\end{array}\right) .
$$

\section{Probabilistic Method to Construct TKDPs}

We will show the existence of a $(t, w, \mathcal{T})-\operatorname{TKDP}(b, n)$, denoted $M=\left(\alpha_{i, j}\right)$, by using the probabilistic method [1]. We would like for the matrix $M$ to have as few rows as possible.

Now we fix a tree $\mathcal{T}$ on a set $V=\{0, \ldots, L-1\}$. Let $S=\{0, \ldots, L-1, \infty\}^{n}$ be the set of all possible rows of length $n$ with symbols from $\{0, \ldots, L-1, \infty\}$. We define a probability distribution $\mathbf{P}$ on the set $S$, and a random variable $X(P, F)$ on $S$ for any disjoint pair of $P \in \mathcal{P}$ and $F \in \mathcal{F}$ as follows:

$$
X(P, F)= \begin{cases}0 & \text { if the row protects } P \text { against } F \\ 1 & \text { otherwise. }\end{cases}
$$

Let $\mathcal{M}_{b \times n}$ be the set of all $b \times n$ matrices with symbols from $\{0, \ldots, L-$ $1, \infty\}$. By the probability distribution $\mathbf{P}$, we pick uniformly at random $b$ rows to construct a matrix in $\mathcal{M}_{b \times n}$. The distribution $\mathbf{P}$ and the random variable $X(P, F)$ naturally define the inherited probability distribution $\mathbf{P}_{b}$ and random variable

$$
X_{b}(P, F)= \begin{cases}0 & \text { if there exists a row that protects } P \text { against } F \\ 1 & \text { otherwise. }\end{cases}
$$

on $\mathcal{M}_{b \times n}$. Then we have

$$
\begin{aligned}
\operatorname{Exp}\left[X_{b}(P, F)\right] & =\operatorname{Pr}\left[X_{b}(P, F)=0\right] \times 0+\operatorname{Pr}\left[X_{b}(P, F)=1\right] \times 1 \\
& =(\operatorname{Pr}[X(P, F)=1])^{b} .
\end{aligned}
$$

Finally, we define a random variable

$$
X=\sum_{P \in \mathcal{P}, F \in \mathcal{F}, P \cap F=\emptyset} X_{b}(P, F) .
$$

If $\operatorname{Exp}[X]<1$, then there exists a $(t, w, \mathcal{T})-\operatorname{TKDP}(b, n)$ in $\mathcal{M}_{b \times n}$. In order to evaluate $\operatorname{Exp}[X]$, we define

$$
\bar{p}_{t, w}=\min _{P \in \mathcal{P}, F \in \mathcal{F}, P \cap F=\emptyset} \operatorname{Pr}[X(P, F)=0] .
$$


Then it is easy to see that

$$
\begin{aligned}
\operatorname{Exp}[X] & =\sum_{P \in \mathcal{P}, F \in \mathcal{F}, P \cap F=\emptyset} \operatorname{Exp}\left[X_{b}(P, F)\right] \\
& =\left(\begin{array}{c}
n \\
t
\end{array}\right)\left(\begin{array}{c}
n-t \\
w
\end{array}\right) \operatorname{Exp}[X(P, F)]^{b} \\
& \leq n^{t+w}\left(1-\bar{p}_{t, w}\right)^{b} .
\end{aligned}
$$

A simple computation shows

$$
b>\frac{(t+w) \ln n}{-\ln \left(1-\bar{p}_{t, w}\right)} \Longrightarrow \operatorname{Exp}[X]<1,
$$

where

$$
\frac{(t+w) \ln n}{-\ln \left(1-\bar{p}_{t, w}\right)} \approx \frac{(t+w) \ln n}{\bar{p}_{t, w}},
$$

for sufficiently small $\bar{p}_{t, w}$. Note that $\bar{p}_{t, w}$ is determined by the probability distribution $\mathbf{P}$ on $S$ and the tree $\mathcal{T}$.

Example 4.1. Let $\mathcal{T}$ be a chain on $n$ vertices. Suppose we define

$$
\begin{aligned}
& \mathbf{P}:\{0, \ldots, n-1\}^{n} \longrightarrow[0,1] \\
& \alpha \mapsto 1 / n \text { ! if } \alpha \text { is a permutation of the } n \text { distinct symbols, } \\
& 0 \text { otherwise. }
\end{aligned}
$$

Since

$$
\bar{p}_{t, w}=\frac{t ! w !}{(t+w) !},
$$

we have a $(t, w, \mathcal{T})-\operatorname{TKDP}(b, n)$ for

$$
b \approx \frac{(t+w)(t+w) ! \ln n}{t ! w !} .
$$

In the following sections, we consider two kinds of probability distributions to generate random rows.

\subsection{Independent Random Selection of Symbols}

In this section, we fix a tree $\mathcal{T}$ on $V=\{0, \ldots, L-1\}$, and find the optimal probability distribution such that each symbol is chosen independently; each symbol $i \in V \cup\{\infty\}$ is selected with probability $p_{i}$, where $0 \leq p_{i} \leq 1$ for $i=0, \ldots, L-1, \infty$, and

$$
p_{0}+\ldots+p_{L-1}+p_{\infty}=1 \text {. }
$$

Then the probability distribution $\mathbf{P}$ on $S=\{0, \ldots, L-1, \infty\}^{n}$ is derived as follows:

$$
\begin{aligned}
\mathbf{P}: S & \longrightarrow[0,1] \\
\alpha & \mapsto \prod_{i=0}^{L-1} p_{i}^{w t_{i}(\alpha)},
\end{aligned}
$$

where $w t_{i}(\alpha)$ is the number of $i$ 's appearing in $\alpha$. Now we have the following lemma. 
Lemma 4.1. Let $\mathbb{P}(v)$ denote the path from the root 0 to $v \in V$ in $\mathcal{T}$ and let $\operatorname{prt}(v)$ denote the parent of $v$. Then we have

$$
\operatorname{Pr}[X(P, F)=0]=\sum_{v \in V}\left(\left(\sum_{i \in \mathbb{P}(v)} p_{i}\right)^{t}-\left(\sum_{i \in \mathbb{P}(\operatorname{prt}(v))} p_{i}\right)^{t}\right)\left(1-\sum_{i \in \mathbb{P}(v)} p_{i}\right)^{w}
$$

for any disjoint $P \in \mathcal{P}$ and $F \in \mathcal{F}$.

Proof. Note that $\operatorname{Pr}[X(P, F)=0]$ is the probability that a row(sequence) protects $P$ against $F$ when it is chosen by the probability distribution $\mathbf{P}$. Suppose that we pick up a sequence $\alpha=\left(\alpha_{1}, \ldots, \alpha_{n}\right)$. If $\alpha$ protects $P$ against $F$, then there exists $j^{*} \in P$ such that $\alpha_{j} \leq \alpha_{j^{*}}$, for all $j \in P$ and $\alpha_{j} \not \alpha_{j^{*}}$, for all $j \in F$. Since the symbol $\alpha_{j^{*}}$ is unique, we can define $\max (\alpha, P)=\alpha_{j^{*}}$ for any sequence $\alpha$ that protects $P$ against $F$.

If $\max (\alpha, P)=v$, then the positions of $P$ should consist of symbols of the path from the root to the vertex $v$, and contain at least one symbol of $v$. On the other hand, the positions of $F$ should contain no symbol from the path. Therefore we have

$$
\operatorname{Pr}[\max (\alpha, P)=v]=\left(\left(\sum_{i \in \mathbb{P}(v)} p_{i}\right)^{t}-\left(\sum_{i \in \mathbb{P}(p r t(v))} p_{i}\right)^{t}\right)\left(1-\sum_{i \in \mathbb{P}(v)} p_{i}\right)^{w},
$$

for every $v \in V$. Now the lemma is true since

$$
\operatorname{Pr}[X(P, F)=0]=\sum_{v \in V} \operatorname{Pr}[\max (\alpha, P)=v] .
$$

By using the above lemma, we can find optimal probability distributions for some special cases.

$\boldsymbol{L}=\mathbf{2}$. We have a unique tree $\mathcal{T}$ on 2 vertices. We would like to maximize the probability to construct a $(t, w, \mathcal{T})-\operatorname{TKDP}(b, n)$, denoted $M=\left(\alpha_{i, j}\right)$. In this model, symbols 0,1 and $\infty$ are selected in a independent random way with probabilities $p_{0}, p_{1}$ and $p_{\infty}$, respectively. By Lemma 4.1 we have

$$
\bar{p}_{t, w}=p_{0}^{t}\left(1-p_{0}\right)^{w}+\left(\left(p_{0}+p_{1}\right)^{t}-p_{0}^{t}\right)\left(1-p_{0}-p_{1}\right)^{w} .
$$

Removing $p_{\infty}$, we have to solve the following problem to find an optimal probability distribution:

$$
\begin{array}{rr}
\text { Maximize } & \bar{p}_{t, w}=p_{0}^{t}\left(1-p_{0}\right)^{w}+\left(\left(p_{0}+p_{1}\right)^{t}-p_{0}^{t}\right)\left(1-p_{0}-p_{1}\right)^{w} \\
\text { subject to } & p_{0}+p_{1} \leq 1, p_{0}, p_{1} \geq 0 .
\end{array}
$$


Example 4.2. Let $t=2, w=1$ and $n=1000$. Then the above problem is reduced to

$$
\begin{array}{rr}
\text { Maximize } & \bar{p}_{2,1}=-p_{0}^{3}-2 p_{0}^{2} p_{1}+p_{0}^{2}-3 p_{0} p_{1}^{2}+2 p_{0} p_{1}-p_{1}^{3}+p_{1}^{2} \\
\text { subject to } & p_{0}+p_{1} \leq 1, p_{0}, p_{1} \geq 0 .
\end{array}
$$

By elementary calculus, we can show that $\bar{p}_{2,1}$ attains its maximum $\bar{p}_{2,1}^{*} \approx 0.204$ when

$$
p_{0}=\frac{12}{23}, \quad p_{1}=\frac{6}{23}, \text { and } p_{\infty}=\frac{5}{23} .
$$

By the condition (1) , there exists a $(2,1, \mathcal{T})$-TKDP $(91,1000)$. On the other hand, if we use a probabilistic method [7] for cover-free families, we can show the existence of a $(2,1)-C F F(130,1000)$ at best. Therefore we have about $40 \%$ improvement in the number of rows.

Star-Like Trees with Many Leaves. Suppose $\mathcal{T}$ is a star-like tree with $a$ leaves (labeled from 1 to $a$ ). We choose each leaf with the same probability $p_{1}$, and set $p_{\infty}=0$ and $t=2$, for simple analysis. Then we have to solve the following problem:

$$
\begin{array}{rr}
\text { Maximize } & \bar{p}_{2, w}=p_{0}^{2}\left(1-p_{0}\right)^{w}+a\left(\left(p_{0}+p_{1}\right)^{2}-p_{0}^{2}\right)\left(1-p_{0}-p_{1}\right)^{w} \\
\text { subject to } & p_{0}+a p_{1}=1, p_{0}, p_{1} \geq 0 .
\end{array}
$$

Put $x=p_{0}$ and $y=a p_{1}$. For a sufficiently large $a$, we have the approximation

$$
\begin{aligned}
\bar{p}_{2, w} & =(1-y)^{2} y^{w}+a\left(\left(x+\frac{y}{a}\right)^{2}-x^{2}\right)\left(y-\frac{y}{a}\right)^{w} \\
& =(1-y)^{2} y^{w}+\left(2 x y+\frac{y^{2}}{a}\right)\left(y-\frac{y}{a}\right)^{w} \\
& \approx\left((1-y)^{2}+2 x y\right) y^{w} \\
& =\left(1-y^{2}\right) y^{w} .
\end{aligned}
$$

Since

$$
\begin{aligned}
\frac{d}{d y}\left(\left(1-y^{2}\right) y^{w}\right) & =(-2 y) y^{w}+w\left(1-y^{2}\right) y^{w-1} \\
& =\left(w-(w+2) y^{2}\right) y^{w-1}
\end{aligned}
$$

we see that $\bar{p}_{2, w}$ attains its (approximate) maximum

$$
\bar{p}_{2, w}^{*} \approx \frac{2}{w+2}\left(\frac{w}{w+2}\right)^{w / 2}
$$

when $y=\sqrt{\frac{w}{w+2}}$, or equivalently,

$$
p_{0}=1-\sqrt{\frac{w}{w+2}}, p_{1}=\frac{1}{a} \sqrt{\frac{w}{w+2}},\left(\text { and } p_{\infty}=0\right) .
$$


By the condition (1), we know there exists a $(2, w, \mathcal{T})-\operatorname{TKDP}(b, n)$ if

$$
b>\frac{1}{2}(w+2)^{2}\left(1+\frac{2}{w}\right)^{w / 2} \ln n,
$$

for sufficiently large $w$. It is instructive to observe that a similar method shows the existence of $(2, w)-\mathrm{CFF}(b, n)$ for

$$
b>\frac{1}{4}(w+2)^{3}\left(1+\frac{2}{w}\right)^{w} \ln n .
$$

\subsection{Random Permutations}

In this section, we fix a probabilistic distribution, and find the optimal tree ordering. We extend Example4.1 to a (general) tree $\mathcal{T}$ on a set $V=\{0, \ldots, L-1\}$ of vertices such that $L+1 \geq n$. We define a probability distribution $\mathbf{P}$ on $S=\{0, \ldots, L-1, \infty\}^{n}$ as follows:

$$
\begin{aligned}
& \mathbf{P}: S \longrightarrow[0,1] \\
& \alpha \mapsto(L+1-n) ! /(L+1) \text { ! if } \alpha \text { is a permutation of an } n \text {-subset of } V \cup\{\infty\} \text {, } \\
& 0 \quad \text { otherwise. }
\end{aligned}
$$

Then we have the following lemma.

Lemma 4.2. Let $m_{d}$ be the number of vertices whose distance from the root is d. Then we have

$$
\operatorname{Pr}[X(P, F)=0]=\frac{\sum_{d \geq t-1} m_{d}\left(\left(\begin{array}{c}
d+1 \\
t
\end{array}\right)-\left(\begin{array}{c}
d \\
t
\end{array}\right)\right)\left(\begin{array}{c}
L-d \\
w
\end{array}\right) t ! w !}{\left(\begin{array}{c}
L+1 \\
t+w
\end{array}\right)(t+w) !}
$$

for any disjoint $P \in \mathcal{P}$ and $F \in \mathcal{F}$.

Example 4.3. Let $t=2, w=1$, and let $\mathcal{T}$ be a binary tree on $L=7$ vertices as seen in Fig. 1(b), Since $m_{0}=1, m_{1}=2$ and $m_{2}=4$, we have

$$
\begin{aligned}
\operatorname{Pr}[X(P, F)=0] & =\frac{2 \cdot\left(\begin{array}{l}
2 \\
2
\end{array}\right)\left(\begin{array}{l}
6 \\
1
\end{array}\right) \cdot 2 !+4 \cdot\left(\left(\begin{array}{l}
3 \\
2
\end{array}\right)-\left(\begin{array}{l}
2 \\
2
\end{array}\right)\right)\left(\begin{array}{l}
5 \\
1
\end{array}\right) \cdot 2 !}{\left(\begin{array}{l}
8 \\
3
\end{array}\right) \cdot 3 !} \\
& =\frac{31}{84} \approx 0.369 .
\end{aligned}
$$

by Lemma 4.2. Therefore, when we choose a sequence from $S=\{0, \ldots, 6, \infty\}^{n}$ by the probability distribution $\mathbf{P}$ (for any $n \leq 8$ ), the sequence protects any two users against another single user with the probability $\approx 0.369$.

Proof. Note that $\operatorname{Pr}[X(P, F)=0]$ is the probability that a row(sequence) protects $P$ against $F$ when it is chosen by the probability distribution $\mathbf{P}$. Suppose that we choose a sequence $\alpha=\left(\alpha_{1}, \ldots, \alpha_{n}\right)$. If $\alpha$ protects $P$ against $F$, then there exists $j^{*} \in P$ such that $\alpha_{j} \leq \alpha_{j^{*}}$, for all $j \in P$ and $\alpha_{j} \nless \alpha_{j^{*}}$, for all $j \in F$. 
Since the symbol $\alpha_{j^{*}}$ is unique, we can define $\max (\alpha, P)=\alpha_{j^{*}}$ for any sequence $\alpha$ that protects $P$ against $F$.

If $\max (\alpha, P)=v$, then the positions of $P$ should consist of symbols of the path from the root to the vertex $v$, and contain exactly one symbol of $v$. On the other hand, the positions of $F$ should contain no symbol from the path. Therefore we have

$$
\operatorname{Pr}[\max (\alpha, P)=v]=\frac{\left(\left(\begin{array}{c}
d+1 \\
t
\end{array}\right)-\left(\begin{array}{c}
d \\
t
\end{array}\right)\right)\left(\begin{array}{c}
L-d \\
w
\end{array}\right) t ! w !}{\left(\begin{array}{c}
L+1 \\
t+w
\end{array}\right)(t+w) !}
$$

where $d \geq t-1$ is the distance from the root to the vertex $v$. If $d<t-1$, then $\operatorname{Pr}[\max (\alpha, P)=v]=0$ since the symbols of $P$ cannot be all distinct. Now the lemma follows since

$$
\operatorname{Pr}[X(P, F)=0]=\sum_{v \in V} \operatorname{Pr}[\max (\alpha, P)=v] .
$$

In order to find an optimal tree which maximizes $\bar{p}_{t, w}$, we have to solve the following optimization problem:

$$
\begin{gathered}
\text { Maximize } \bar{p}_{t, w}=\frac{\sum_{d \geq t-1} m_{d}\left(\left(\begin{array}{c}
d+1 \\
t
\end{array}\right)-\left(\begin{array}{c}
d \\
t
\end{array}\right)\right)\left(\begin{array}{c}
L-d \\
w
\end{array}\right) t ! w !}{\left(\begin{array}{c}
L+1 \\
t+w
\end{array}\right)(t+w) !} \\
\text { subject to } \sum_{d=1}^{D} m_{d}=L-1, \\
D, m_{1}, \ldots m_{D} \in \mathbb{Z}^{+} .
\end{gathered}
$$

Let

$$
\begin{aligned}
C_{d} & =\frac{\left(\left(\begin{array}{c}
d+1 \\
t
\end{array}\right)-\left(\begin{array}{l}
d \\
t
\end{array}\right)\right)\left(\begin{array}{c}
L-d \\
w
\end{array}\right) t ! w !}{\left(\begin{array}{c}
L+1 \\
t+w
\end{array}\right)(t+w) !} \\
& =\left(\frac{(d+1) !}{(d+1-t) !}-\frac{d !}{(d-t) !}\right) \frac{(L-d) !}{(L-d-w) !} \cdot \frac{(L+1-t-w) !}{(L+1) !} \\
& =\frac{t \cdot d !}{(d+1-t) !} \cdot \frac{(L-d) !}{(L-d-w) !} \cdot \frac{(L+1-t-w) !}{(L+1) !} .
\end{aligned}
$$

$C_{d}$ increases at $d$ (as a function of $d$ ) if

$$
C_{d-1}<C_{d} \Leftrightarrow d<\frac{(L+1)(t-1)}{t+w-1} .
$$

Therefore $C_{d}$ attains its maximum at

$$
d^{*}=\left\lceil\frac{(L+1)(t-1)}{t+w-1}-1\right\rceil,
$$


and we can solve the problem (2) with an optimal solution

$$
\left(D^{*}, m_{1}^{*}, \ldots, m_{D}^{*}\right)=(d^{*}, \overbrace{1, \ldots, 1}^{d^{*}-1}, L-d^{*}),
$$

and the optimal value

$$
\begin{aligned}
\bar{p}_{t, w, L}^{*} & =\frac{\left.\sum_{d=t-1}^{d^{*}} m_{d}^{*}\left(\begin{array}{c}
d+1 \\
t
\end{array}\right)-\left(\begin{array}{c}
d \\
t
\end{array}\right)\right)\left(\begin{array}{c}
L-d \\
w
\end{array}\right) t ! w !}{\left(\begin{array}{c}
L+1 \\
t+w
\end{array}\right)(t+w) !} \\
& =\frac{\sum_{d=t-1}^{d^{*}} m_{d}^{*}((d+1) ! /(d+1-t) !-d ! /(d-t) !)((L-d) ! /(L-d-w) !)}{(L+1) ! /(L+1-t-w) !} \\
& =\frac{(L+1-t-w) !}{(L+1) !} \sum_{d=t-1}^{d^{*}} \frac{m_{d}^{*} t \cdot d !}{(d+1-t) !} \cdot \frac{(L-d) !}{(L-d-w) !} \\
& \geq \frac{(L+1-t-w) !}{(L+1) !} \cdot \frac{\left(L-d^{*}\right) t \cdot d^{*} !}{\left(d^{*}+1-t\right) !} \cdot \frac{\left(L-d^{*}\right) !}{\left(L-d^{*}-w\right) !} \\
& =\frac{\left(L-d^{*}\right) t \cdot \overbrace{d^{*}\left(d^{*}-1\right) \ldots\left(d^{*}-t+2\right)}^{(L-1)} \cdot \overbrace{\left(L-d^{*}\right)\left(L-d^{*}-1\right) \ldots\left(L-d^{*}-w+1\right)}^{(L+1) L \ldots(L-t-w+2)}}{t+1} \\
& =w \Omega\left(\frac{t ! w !}{(t+w) !}\right),
\end{aligned}
$$

since

$$
\frac{d^{*}}{L} \approx \frac{t}{t+w}
$$

for a sufficiently large $L$.

The optimal tree looks like a claw, as seen in Fig. 3. Now we conclude that

1. the probability $\bar{p}_{t, w}$ based on the optimal tree is greater than the one based on a chain (asymptotically) by a factor of $w$, and

2. the maximum hash depth applied to a secret seed is $d^{*}=\lceil(L+1)(t-1) /(t+$ $w-1)-17$, which is relatively small for a large $w$.

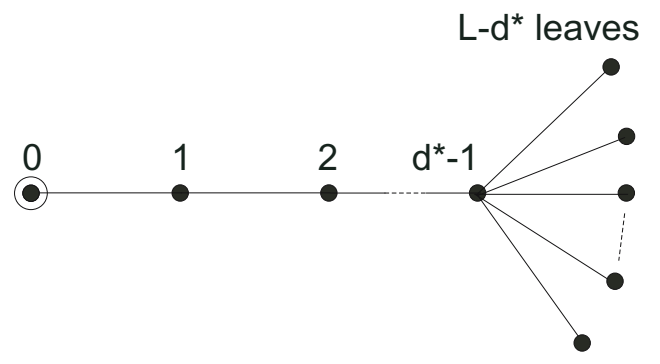

Fig. 3. Optimal tree 
Example 4.4. Let $n=L=1000, t=2$ and $w=100$. Then we have $d^{*}=$ $\left\lceil\frac{1001}{101}-1\right\rceil=9$, and $\bar{p}_{t, w, L}^{*} \geq 0.00764$, while $\frac{t ! w !}{(t+w) !}=0.00019$. By the condition (1), there exists a $\left(2,100, \mathcal{T}^{*}\right)-\operatorname{TKDP}(91872,1000)$, where $\mathcal{T}^{*}$ is a claw of depth $d^{*}=9$.

\section{Conclusion}

We presented a new framework of tree-based key distribution pattern (TKDP). We constructed TKDPs from cover-free families. We note that other combinatorial structures such as orthogonal arrays, ordered designs and covering arrays also yield TKDPs. (This will be addressed in later work.) We showed the existence of TKDPs by the probabilistic method. Furthermore, we reduced the upper bounds on the minimum number of rows of $(t, w, \mathcal{T})$-TKDPs, which are obtained from probabilistic methods, asymptotically by a factor of $w$ as compared to Leighton and Micali's schemes by choosing optimal trees $\mathcal{T}$ instead of chains. The TKDP-based schemes are expected to have applications to sensor networks since they involve only hash computations in the establishment of pairwise keys, which are known to be more energy-efficient than RSA or elliptic curve operations.

\section{References}

1. N. Alon and J. Spencer. The Probabilistic Method, Wiley, New York, 1992.

2. N. Attrapadung, K. Kobara and H. Imai. Sequential key derivation patterns for broadcast encryption and key predistribution schemes, Lecture Notes in Computer Science, 2894 (2003), 374-391 (Advances in Cryptology - ASIACRYPT '03).

3. R. Blom. An Optimal Class of Symmetric Key Generation Systems, Lecture Notes in Computer Science, 209 (1985), 335-338 (Advances in Cryptology - EUROCRYPT '84).

4. C. Blundo, A. De Santis, A. Herzberg, S. Kutten, U. Vaccaro and M. Yung. Perfectly-secure key distribution for dynamic conferences, Lecture Notes in Computer Science, 740 (1993), 148-168 (Advances in Cryptology - EUROCRYPT '92).

5. H. Chan, A. Perrig, and D. Song. Random Key Predistribution Schemes for Sensor Networks, In IEEE Symposium on Research in Security and Privacy, 197-213, May 2003.

6. C.J. Colbourn and J.H. Dinitz, editors. The CRC Handbook of Combinatorial Designs, CRC Press, Boca Raton, 1996.

7. M. Dyer, T. Fenner, A. Frieze and A. Thomason, On key storage in secure networks. Journal of Cryptology, 8 (1995), 189-200.

8. L. Eschenauer and V.D. Gligor. A Key-Management Scheme for Distributed Sensor Networks, In Proceedings of the 9th ACM conference on Computer and communications security, 41-47, November 2002.

9. J. Lee and D.R. Stinson. A combinatorial approach to key predistribution for distributed sensor networks. the IEEE Wireless Communications and Networking Conference, CD-ROM, 2005, paper PHY53-06, 6-11, http:// www.cacr.math.uwaterloo.ca/ dstinson/pubs.html. 
10. J. Lee and D.R. Stinson. Deterministic key predistribution schemes for distributed sensor networks. Lecture Notes in Computer Science 3357 (2004), 294-307 (SAC 2004 Proceedings).

11. T. Leighton and S. Micali, Secret-key agreement without public-key cryptography, Lecture Notes in Computer Science, 773 (1994), 456-479 (Advances in Cryptology CRYPTO '93).

12. D. Liu and P. Ning, Establishing Pairwise Keys in Distributed Sensor Networks, In Proceedings of the 10th ACM Conference on Computer and Communications Security (CCS), 52-61, October 2003.

13. C.J. Mitchell and F.C. Piper, Key storage in secure networks. Discrete Applied Mathematics, 21 (1988), 215-228.

14. C. Padró, I. Grasia, S.M. Molleví and P. Morillo, Linear key predistribution schemes. Designs, Codes and Cryptography, 25 (2002), 281-298.

15. M. Ramkumar and N. Memon, HARPS-Hashed Random Preloaded Subset Key Distribution. Cryptology ePrint Archive, Report 2003/170 (2003), http://eprint.iacr.org/2003/170.

16. D.R. Stinson and Tran van Trung, Some new results on key distribution patterns and broadcast encryption. Designs, Codes and Cryptography, 14 (1998), 261-279.

17. D.R. Stinson and R. Wei, Generalized cover-free families. Discrete Mathematics, 279 (2004), 463-477.

18. R. Wei, On cover-free families. Discrete Mathematics, to appear. 